\title{
Ex vivo hepatectomy and partial liver autotransplantation for hepatoid adenocarcinoma: A case report
}

\author{
FU-QIANG WANG ${ }^{1 *}$, QIAN LU ${ }^{2 *}$, JUN YAN $^{2}$, YOU-YUAN PENG ${ }^{1}$, CHENG-RONG XIE $^{1}$, YONG-JIE SU $^{1}$, \\ JIAN-YIN ZHOU ${ }^{1}$, BO-LIANG WANG ${ }^{3}$, WEN-XIU ZHAO ${ }^{1}$, XIAO-MIN WANG $^{1}$, PING BIE ${ }^{2}$ and ZHEN-YU YIN ${ }^{1}$ \\ ${ }^{1}$ Department of Hepatobiliary Surgery, Zhongshan Hospital of Xiamen University, \\ Fujian Provincial Key Laboratory of Chronic Liver Disease and Hepatocellular Carcinoma, Xiamen, Fujian; \\ ${ }^{2}$ Department of Hepatobiliary Surgery, Southwest Hospital, The Third Military Medical University, Chongqing; \\ ${ }^{3}$ School of Information Science and Technology, Xiamen University, Xiamen, Fujian, P.R. China
}

Received June 23, 2014; Accepted February 26, 2015

DOI: $10.3892 / 01.2015 .3041$

\begin{abstract}
We previously reported the case of a 56-year-old male who underwent surgical treatment for gastric hepatoid adenocarcinoma and splenic metastasis. The present study reports the case of the same patient who underwent successful ex vivo hepatectomy and partial liver autotransplantation. Computed tomography scans demonstrated that the tumor was located in the left and caudate lobes of the liver, with hepatic vein and inferior vena cava involvement, and right portal vein compression. To clarify the association between the vessels and the tumor, a three-dimensional imaging technique was used to reconstruct the liver architecture. During the surgery, the whole liver was removed with the retrohepatic vena cava, which was replaced with a prosthetic graft without a veno-venous bypass; a portacaval shunt for the anhepatic phase was performed simultaneously. The surgery lasted $9 \mathrm{~h}$, and the anhepatic phase lasted $4 \mathrm{~h}$. Blood loss during the surgery was recorded at $1,500 \mathrm{ml}$. The time taken for recovery was 21 days post-surgery, and at 20 months, the patient was disease-free. To the best of our knowledge, this is the first case of ex vivo liver resection performed for hepatic metastasis of hepatoid adenocarcinoma to be reported.
\end{abstract}

\section{Introduction}

Ex situ liver surgery is a novel technique for the treatment of complicated liver tumors that are unresectable by conventional

Correspondence to: Professor Zhen-Yu Yin, Department of Hepatobiliary Surgery, Zhongshan Hospital of Xiamen University, Fujian Provincial Key Laboratory of Chronic Liver Disease and Hepatocellular Carcinoma, 209 South Hubin Road, Xiamen, Fujian 361004, P.R. China

E-mail: yinzy@xmu.edu.cn

*Contributed equally

Key words: ex vivo hepatectomy, hepatoid adenocarcinoma, hepatocellular carcinoma methods. Since it was first described by Pichlmayr et al (1) in 1988, ex situ surgery has increased the survival expectancy of a number of patients with unresectable liver tumors. Liver autotransplantation, which has developed from precise liver resection and liver transplantation, has expanded the surgical indications for primary and secondary liver tumors or neoplasms involving the inferior vena cava (IVC) (2).

Hepatoid adenocarcinoma (HAC) is a rare and specific type of extrahepatic adenocarcinoma that morphologically mimics hepatocellular carcinoma (HCC). The most common features of HAC are elevated levels of serum $\alpha$-fetoprotein (AFP) and carcinoembryonic antigen (CEA), as well as tissue immunoreactivity for AFP. HAC is most commonly detected in the stomach (3). Since the tumor frequently occurs with liver and lymph node metastases, the prognosis is poor (4). It is difficult to differentiate the hepatic metastasis of HAC from HCC due to their similar clinicopathological characteristics. The treatment of HAC is more difficult when the tumor has metastasized to the liver, and surgical treatment of the metastases is rarely undertaken. In a previous study, we reported the case of a patient with metastatic splenic $\alpha$-fetoprotein-producing adenocarcinoma who had undergone splenectomy (5). The present study reports the case of the same patient who underwent a ex vivo liver resection and partial liver autotransplantation for the treatment of hepatic metastasis from HAC. Written informed consent was obtained from the patient.

\section{Case report}

Patient. In January 2004, a 56-year-old male was admitted to Zhongshan Hospital of Xiamen University (Xiamen, China) due to epigastralgia and melena, with no relevant past medical history. A physical examination revealed no abnormalities. The serum was negative for $\alpha$-fetoprotein, carcinoembryonic antigen, cancer antigen (CA)125, CA199, CA153 and CA724 and positive for hepatitis B surface antigen. Endoscopic examination revealed an infiltrating ulcerative tumor with unclear boundaries, which indicated a diagnosis of advanced gastric cancer of Borrmann type III. A radical gastrectomy was performed in January 2004. Post-operatively, the patient received chemotherapy with three 28 day cycles of 

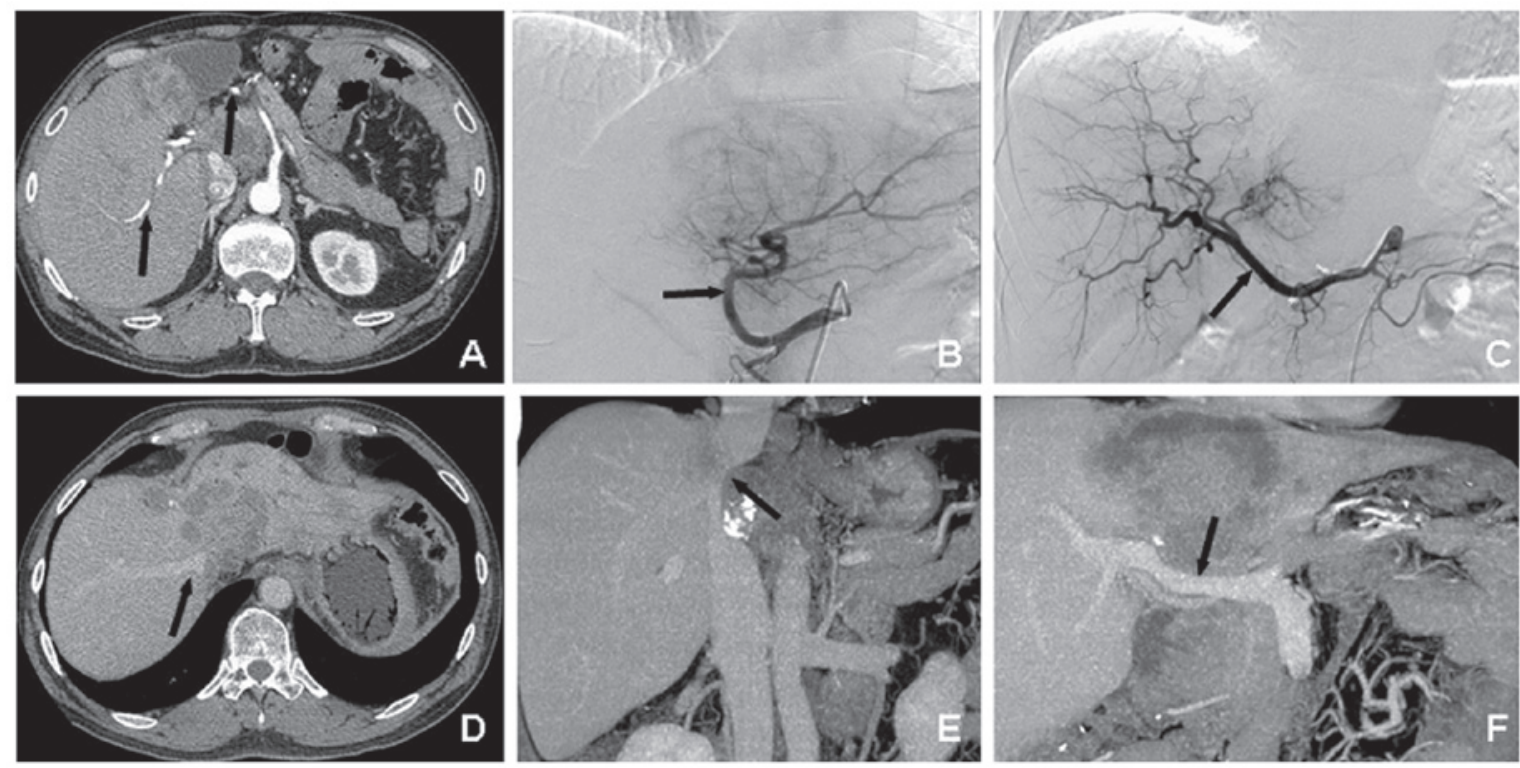

Figure 1. Computed tomography scan and arterial angiography. (A) The right hepatic artery and the main hepatic artery can be observed (arrows) (B) Angiography revealing the left hepatic artery (arrow) from the celiac artery. (C) The right hepatic artery (arrow) from the superior mesenteric artery is evident. (D) The right hepatic vein (arrow) and compressed inferior vena cava (IVC) can be observed. (E) Tumor compression of the IVC (arrow). (F) The right portal vein close to the tumor and the left portal vein are involved.
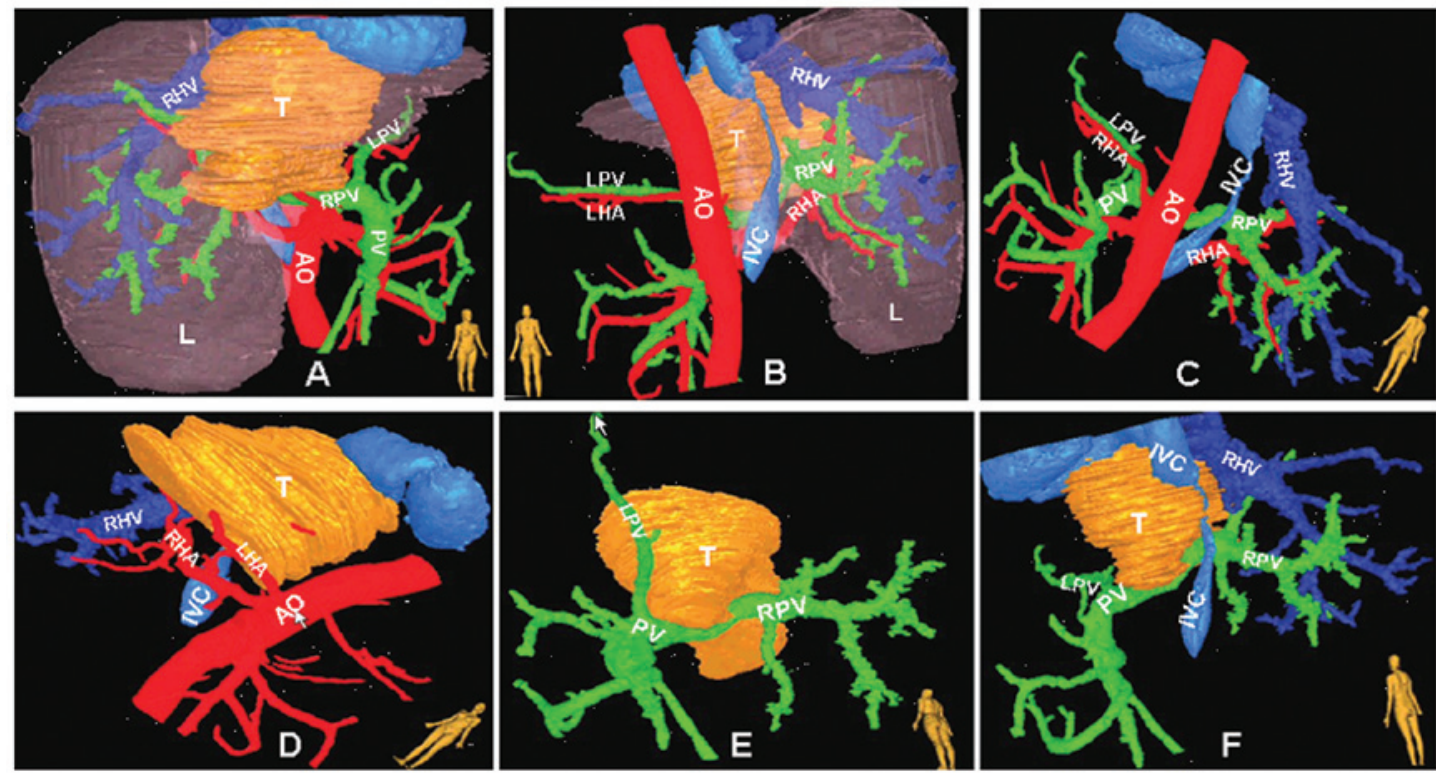

Figure 2. Three-dimensional imaging of the liver illustrates the association of the tumor with the vasculature, using the software we developed. (A, B, D-F) Tumor (yellow), (A-D) hepatic artery (red), (C and F) hepatic vein and IVC (blue), (A, E and F) portal vein (green). IVC, inferior vena cava; T, tumor; L, liver; PV, portal vein; RPV, right portal vein; LPV, left portal vein; AO, aorta; HA, hepatic artery; RHA, right hepatic artery; LHA, left hepatic artery.

5-fluorouracil $\left(500 \mathrm{mg} / \mathrm{m}^{2}\right.$, days $\left.1-5\right)+$ cisplatin $\left(15-20 \mathrm{mg} / \mathrm{m}^{2}\right.$, days 1-5) + leucovorin (200-400 mg/m², days 1-2). In April 2009, the patient was once again admitted to Zhongshan Hospital of Xiamen University due to a splenic neoplasm detected on computed tomography (CT) scans. The serum AFP value was $732 \mathrm{ng} / \mathrm{ml}$ (normal range, $0-25 \mathrm{ng} / \mathrm{ml}$ ) and the CEA level was $1,049 \mathrm{ng} / \mathrm{ml}$ (normal range, $0-3.4 \mathrm{ng} / \mathrm{ml}$ ). No local recurrence of the tumor was found on endoscopy. A splenectomy was subsequently performed. Histopathological examination indicated splenic metastasis from HAC; the immunohistochemical stain was positive for AFP. Intraperitoneal chemotherapy with fluorouracil $\left(1,000 \mathrm{mg} / \mathrm{m}^{2}\right)$ was administered for 5 days post-operatively. However, in July 2009, a positron emission tomography scan showed liver metastases. The patient underwent transcatheter arterial chemoembolization (TACE) and radiofrequency ablation, but the levels of AFP and CEA progressively increased, predicting a poor outcome. The patient received targeted therapy with sorafenib $(0.4 \mathrm{~g}$, twice daily) for two weeks, but could not tolerate further treatment due to adverse reactions. The serum AFP and CEA levels rose to 557 and $609 \mathrm{ng} / \mathrm{ml}$, respectively, in March 2012. An enhanced CT scan revealed multiple metastases in the left and caudate 

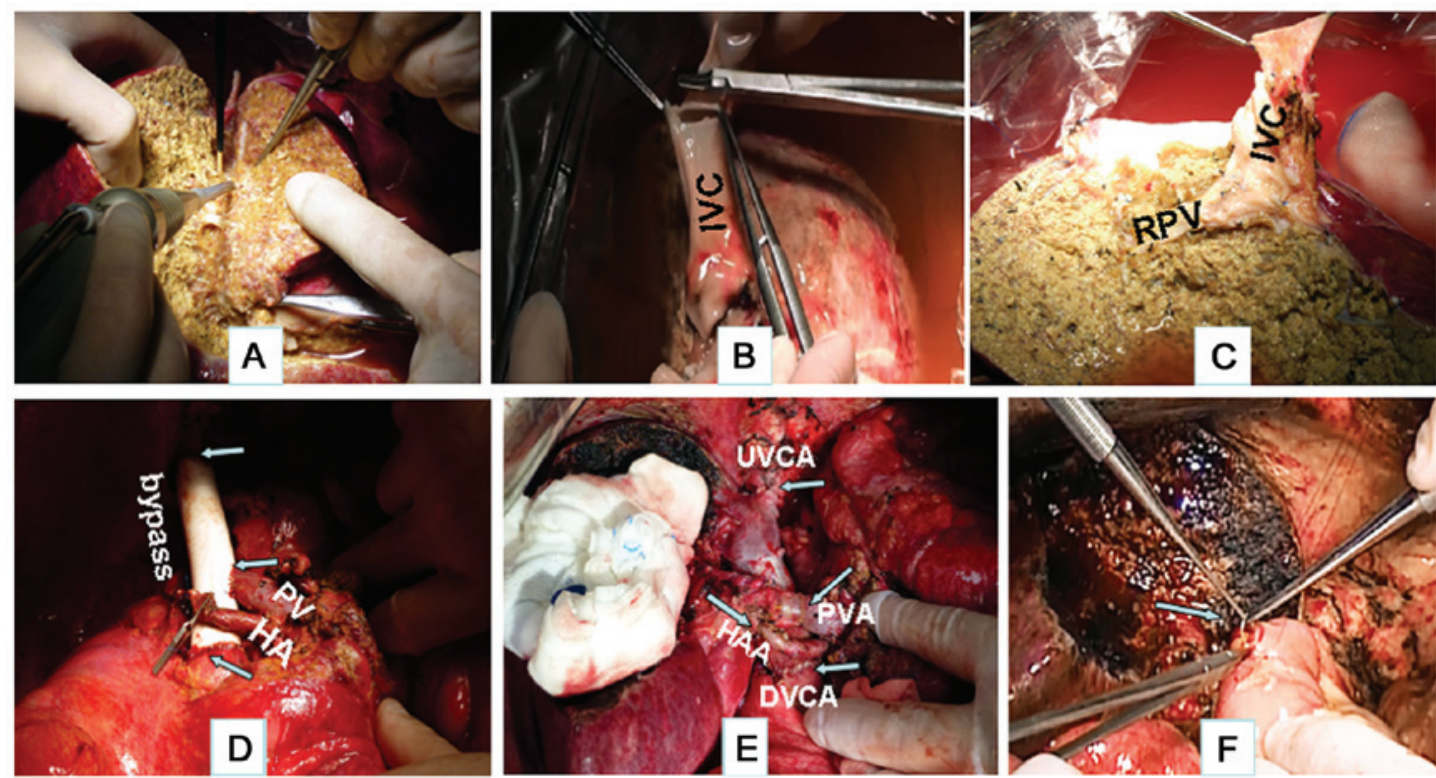

Figure 3. (A) Hepatic resection with the Cavitron Ultrasonic Surgical Aspirator and bipolar coagulation. (B) The liver was placed in an ice bath during resection and reconstruction of the IVC. (C) The right portal vein (RPV) and the inferior vena cava (IVC) are evident in the liver section after the tumor has been removed. (D) The IVC has been replaced with a $20-\mathrm{mm}$ ringed graft, and a temporary portacaval shunt has been constructed. (E) Partial liver reimplantation and anastomosis was performed in the excised IVC, portal vein and hepatic artery. (F) The bile duct (arrow) was anastomosed to the intestine through a Roux-en-Y hepatojejunostomy. HA, hepatic artery; PV, portal vein; UVCA, suprahepatic inferior vena cava anastomosis; PVA, portal vein anastomosis; DVCA, subhepatic inferior vena cava anastomosis; HAA, hepatic artery anastomosis.

lobes of the liver, with compression of the portal vein and IVC (Fig. 1).

Pre-operative serum liver function tests were normal. The indocyanine green clearance test (ICG15) measurement of hepatic reserve function was $5.6 \%$ (normal value, $<10 \%$ ). Reconstruction of the liver with CT scan images using a three-dimensional imaging technique, which we developed (6), is illustrated in Fig. 2.

\section{Surgery}

Intraoperative evaluation. A liver examination by ultrasonography was performed to confirm the location and size of the lesions. A $13 \times 10 \times 10-\mathrm{cm}$, irregular lesion was found in the left and caudate lobes of the liver. The retrohepatic IVC was fully involved. The right hepatic vein was free of tumor. Skeletonization of the hepatoduodenal ligament was performed. The left hepatic artery originated from the celiac axis and supplied the tumor, and the right hepatic artery originated from the superior mesenteric artery.

Total liver resection. The distal segment of the common bile duct was invaded by the tumor, so it was removed following transection of the common duct at the level of cystic duct entry. The hepatic ligaments were freed to expose the IVC and second porta hepatis, revealing that the retrohepatic IVC was three-fourths encircled by the lesion. Once all the vessels had been transected, the liver was removed and placed in an ice bath. Histidine-tryptophan-ketoglutarate solution at $4^{\circ} \mathrm{C}$ was infused via the portal vein and hepatic artery (Fig. 3B).

Temporary portacaval shunt. The IVC was quickly replaced with a $20-\mathrm{mm}$ ringed prosthetic graft, and the portal vein was anastomosed to the graft to reconstitute blood flow (Fig. 3D).

Ex vivo liver resection. The Cavitron Ultrasonic Surgical Aspirator (CUSA; Sonoca 300; Söring GmbH, Quickborn,
Germany) was used to resect the hepatic tissues, and bipolar coagulation was used for disconnecting the small vessels. The big vessels and bile ducts were sutured with 5-0 Prolene. The liver, including the left and caudate lobes, was resected. Perforations of the IVC caused by stripping the tumor from it were sutured with 6-0 Prolene (Fig. 3A and C).

Liver reimplantation. The remaining liver was reimplanted following perfusion via the portal vein with $2 \%$ albumin in Ringer's lactate solution. The suprahepatic and infrahepatic IVC were reconnected by end-to-end anastomosis with 6-0 Prolene. The main portal vein was anastomosed to the right portal vein with 7-0 Prolene. The right hepatic artery was reconnected by end-to-end anastomosis with 7-0 Prolene. Following reconstruction of all the vessels, hemostasis was complete. The bile duct was reconstructed through a roux-en-Y hepatojejunostomy (Fig. 3E and F).

\section{Results}

Surgical summary. The surgery lasted $9 \mathrm{~h}$, with an anhepatic period of $4 \mathrm{~h}$. Blood loss during the surgery was $1,500 \mathrm{ml}$, and four units of packed red cells and $400 \mathrm{ml}$ of fresh-frozen plasma were administered. The patient was cared for in the intensive care unit for three days after the surgery, with no complications post-operatively. The alanine aminotransferase level rose to $620 \mu / 1$ and returned to normal levels seven days later. The patient was discharged on post-operative day 21 .

Pathological examination. Gross liver metastasis from the hepatoid adenocarcinoma is illustrated in Fig. 4A. The majority of the tumor was composed of scattered large pleomorphic or multinucleated giant cells. The cells were markedly atypical, with eosinophilic cytoplasm and round 

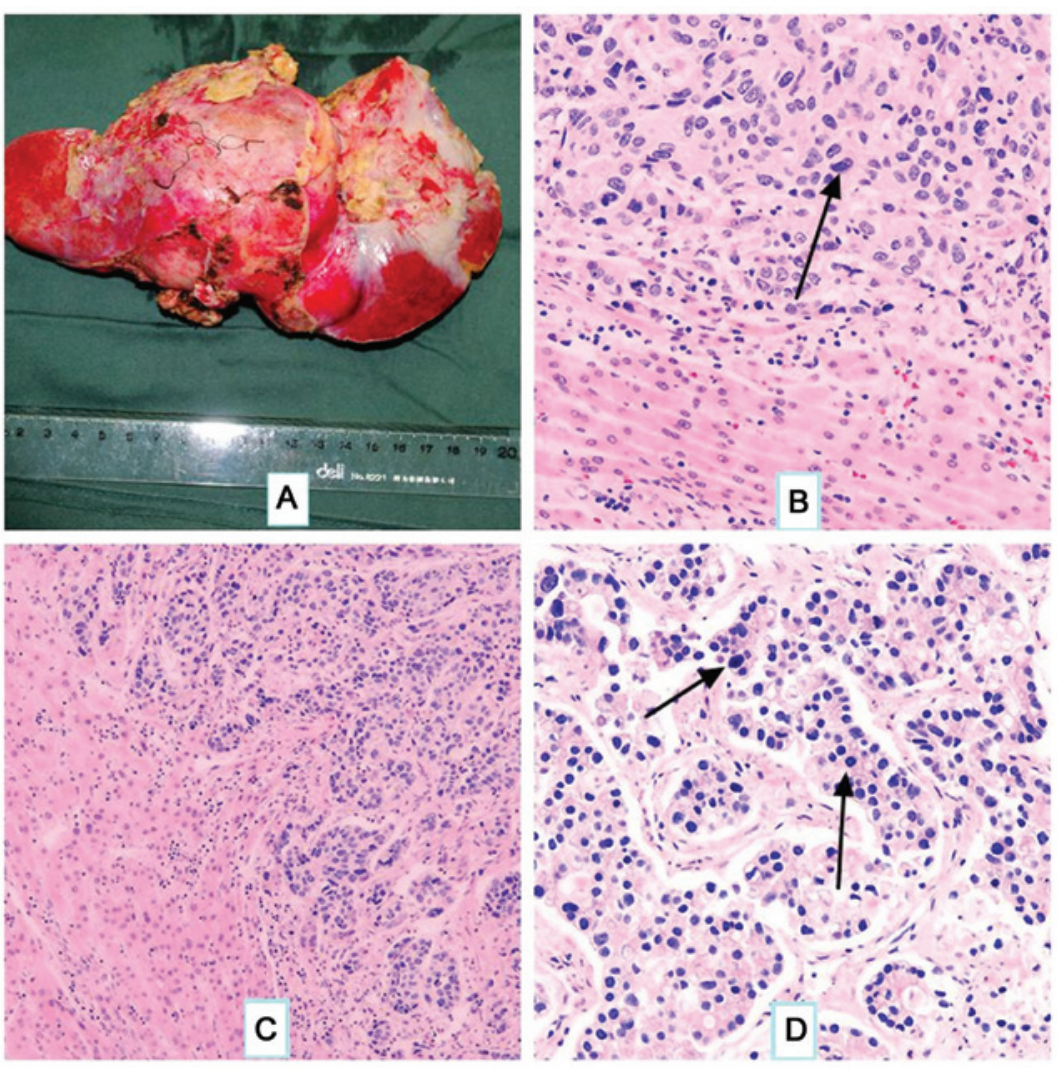

Figure 4. (A) Gross illustration of hepatic metastases. (B-D) Histopathological features of the tumor. (B) Tumor cells are arranged in nests, which are similar to those found in primary liver cancer tissue. 1-2 large and marked nucleoli (arrow) are present in the center of the cells (stain, hematoxylin and eosin; magnification, x200). (C) The tumor cells are arranged in a tubular pattern, similar to those found in hepatocellular carcinoma, and numerous blood sinuses are evident (stain, hematoxylin and eosin; magnification, x100). (D) Tumor cells are arranged with cancer adenoids, with scattered multinucleated giant cells (arrows). Nuclear division can be easily observed (stain, hematoxylin and eosin; magnification, x200).

nuclei, occasionally exhibiting marked nucleoli (Fig. 4B). The tumor cells were arranged in a tubular pattern (Fig. 4C) and nuclear division was evident (Fig. 4D).

Follow-up. The patient remains alive 20 months post-operatively, with normal AFP and CEA levels, and no evidence of recurrent disease.

\section{Discussion}

Bourreille et al (7) first reported an AFP-producing gastric tumor in 1970. Ishikura et al (8) proposed the term HAC of the stomach for this type of primary gastric carcinoma in 1985. The stomach is the most common site of origin of HAC (9), but HAC has been reported in other organs, including the bladder, lungs, pancreas, colon, gall bladder, ovaries and uterus. The reported incidence of HAC is $1.3-15 \%$ of all gastric carcinomas (10). As HAC is relatively rare, the pre-operative diagnostic rate is low, and the diagnosis is usually made through post-operative pathological examination.

It is difficult to differentiate HCC resulting from hepatic metastases from HAC. One previous study (11) reported that the tumors have similar enhancement on CT and magnetic resonance imaging, so it is not easy to differentiate them on the basis of imaging findings. HAC is a relatively rare disease, while liver cirrhosis and chronic hepatitis infection can usually be found among patients with HCC, therefore, the differential diagnosis should be based on the medical history, dynamic image studies and liver biopsy. The proportion of HAC with a positive CEA stain is $>75 \%$, so high levels of serum AFP and CEA together should prompt suspicion of HAC. The patient in the present study had a history of hepatitis B infection and an elevated serum AFP level pre-operatively, therefore, the mass could have been misdiagnosed as HCC. However, the patient also had a history of HAC, had undergone a previous splenic metastasis tumor resection and presented with significant increases in serum CEA level; accordingly, a diagnosis of liver metastasis from HAC was formed.

A number of studies have reported that the prognosis of HAC is extremely poor even when discovered at an early stage. Motoyama et al (4) attributed the poor prognosis of HAC to the frequent occurrence of liver or lymph node metastases. Metastasis of the liver can occur within a year post-operation, even if no metastasis is found pre-operatively. Chang et al (12) reported that the majority of patients in their series, including three who underwent radical surgery for early gastric cancer, succumbed to liver metastasis within two years. Thus, liver metastasis is the main factor affecting the prognosis of $\mathrm{HAC}$, and the close observation and long-term follow-up of patients is required.

Holistic therapy based on surgery is the optimal treatment method for HAC, and surgical treatment for HAC with liver metastasis may still be valuable. the patient in the present case patient developed splenic and hepatic metastases five years after radical resection and chemotherapy for HAC. Metastases 
of HAC to the spleen are believed to be unusual, but a splenectomy was performed on the present patient, as there was no endoscopic evidence of recurrence of the gastric tumor (5). The hepatic metastatic carcinoma was rapidly growing, with invasion of the IVC and the second porta hepatis. The patient's response to TACE was extremely poor. The IVC was stenotic due to the tumor, so surgery was considered as the only option for a cure.

In recent years, with a better understanding of the vascular and segmental anatomy of the liver through the experience of liver transplantation, the results of liver surgery have rapidly improved. Liver tumor metastasis to the vena cava does not obviate the requirement for liver resection (2). Ex vivo liver resection is a novel surgical technique mainly used for large liver tumors located in the dorsum of the liver with involvement of the hepatic veins or the retrohepatic IVC (2). Pichlmayr et al (1) first reported the surgery in 1988 and subsequently performed 11 procedures. This approach has now become recognized and is promoted worldwide. Ex vivo liver resection has increased the resectability rate in patients with advanced tumors, provided a partial solution for bleeding and long-term blocking in certain liver tumor resections, and avoided the requirement for liver transplantation in specific patients. The technique can also be applied to cases of hilar cholangiocarcinoma and complex liver trauma. The present patient presented with liver metastases invading the hepatic vein and retrohepatic IVC, so the tumor was unresectable with conventional surgery. For achieving $\mathrm{R} 0$ resection, ex vivo liver resection appears to be suitable, as it provides enough time for the completion of complex resections and vascular reconstructions in a bloodless environment, thereby reducing the risk of bleeding. Complete pre-operative assessments of liver function and reserve are essential. A computer-aided pre-operative planning system for liver surgery based on CT images has been used to define the association between the tumor and blood vessels (13), and a simulation resection can be used to evaluate the resection range and residual liver volume.

Maintaining a stable hemodynamic status and avoiding ischemia-reperfusion injury are two prominent considerations for this surgery. Certain studies recommend the use of veno-venous bypass during the anhepatic period. The aim of this technique is to reduce hemodynamic instability, ensure splanchnic decompression, and minimize venous hypertension and intestinal edema (14). However, the ex situ ex vivo procedure is associated with complications, including air embolism, thromboembolic events and mechanical injury due to global capillary leak, which can lead to severe post-operative water, electrolyte and acid-base imbalances (15). Thus, the present study used a vascular prosthesis to reconstitute blood flow in the portal vein and IVC to ensure the stability of general circulation, lessen ischemia-reperfusion injury, and avoid acid-alkali and electrolyte imbalances. Wen et al (16) recommended construction of a temporary portacaval shunt to improve hemodynamic status, reduce the requirement for intraoperative blood transfusion and preserve renal function. The shunt can also permit enough time for the completion of the ex vivo liver resection. Compared with the traditional veno-venous bypass technology, this procedure has a shorter surgical duration and anhepatic phase. Zhang et al (17) reported three cases of ex-vivo hepatectomy using this procedure and found no post-operative hepatic dysfunction resulting from the delayed liver hemoperfusion; thus, it was suggested that this method is feasible and safe, with a shortened surgical duration, and less intraoperative bleeding and reperfusion injuries. Gringeri et al (18) performed a temporary portacaval shunt with a stretch of aortic graft to replace the caval vein in a porcine model; the temporary shunt ensured hemodynamic stability during the anhepatic phase and lengthened the portal vein, which facilitated construction of a good anastomosis.

The amount of time that the liver can safely tolerate warm ischemia appears to be limited, so hypothermic perfusion has been considered. The advantage of this technique is that the low temperature increases the tissue tolerance to ischemia. Hannoun et al (19) suggested that hypothermia facilitated the bloodless transection of liver parenchyma and increased the chance of resectability for advanced tumors. The histidine-tryptophan-ketoglutarate solution is better than the University of Wisconsin preservation solution, as it minimizes the risk of cardiocirculatory complications following reperfusion (20). The patient in the present study had a good recovery, without the complications of renal insufficiency and heart failure. At the hypothermic perfusion stage, CUSA and bipolar coagulation were used for transection of the liver parenchyma; thus, the small vessels and bile ducts could be separated during a bloodless condition to prevent bleeding and leakage following re-perfusion. Once all the vessels had been reconstructed, implantation of the remnant liver and anastomosis of vessels was performed exactly as in living donor transplantation. The patient presented with hepatic metastases from HAC, so lymphatic and connective tissue from the hepatoduodenal ligament were removed, which could have compromised the blood supply of the bile duct. Consequently, partial resection of the bile duct was performed and the bile flow was reconstituted with a hepatic-jejunostomy.

Ex situ ex vivo surgery should only be performed in specialized centers, where surgeons are familiar with complex hepatobiliary surgery and liver transplantation. This technique may be the last resource for otherwise unresectable malignancy (14), but the associated morbidity and mortality remain high, and tumor recurrence is a major problem. Raab et al (21) also recommended that the ex situ procedure be used only in specialized centers with extensive experience in both conventional liver surgery and transplantation.

$\mathrm{HAC}$ is prone to liver and lymph node metastasis, which predicts high malignancy and a poor prognosis, but this does not mean that there is no chance of treatment with a good prognosis. Ex situ liver surgery may be the only effective treatment for patients with liver tumors that cannot be resected by traditional procedures. The surgery offers a chance for hepatectomy of the primary and secondary liver tumors, even with hepatic vein and IVC infiltration within the tumor. The safety and efficacy of this technique requires verification through further clinical studies in practice.

\section{Acknowledgements}

This study was supported by grants from the National Natural Science Foundation of China (grant nos. 81172286, 81372618 and 61327001) and the National Key Sci-Tech Special Project of China (grant no. 2012ZX10002-011-005c). 


\section{References}

1. Pichlmayr R, Bretschneider HJ, Kirchner E, et al: Ex situ operation on the liver. A new possibility in liver surgery. Langenbecks Arch Chir 373: 122-126, 1988 (In German).

2. Hemming AW, Mekeel KL, Zendejas I, Kim RD, Sicklick JK and Reed AI: Resection of the liver and inferior vena cava for hepatic malignancy. J Am Coll Surg 217: 115-125, 2013.

3. Nagai E, Ueyama T, Yao T and Tsuneyoshi M: Hepatoid adenocarcinoma of the stomach. A clinicopathologic and immunohistochemical analysis. Cancer 72: 1827-1835, 1993.

4. Motoyama T, Aizawa K, Watanabe H, Fukase M and Saito K: alpha-Fetoprotein producing gastric carcinomas: a comparative study of three different subtypes. Acta Pathol Jpn 43: 654-661, 1993.

5. Deng Z, Yin Z, Chen S, Peng Y, Wang F and Wang X: Metastatic splenic $\alpha$-fetoprotein-producing adenocarcinoma: report of a case. Surg Today 41: 854-858, 2011.

6. Song X, Cheng M, Wang B, Huang S and Huang X: Computeraided preoperative planning for liver surgery based on CT images. Procedia Engineering 24: 133-137, 2011.

7. Bourreille J, Metayer P, Sauger F, Matray F and Fondimare A: Existence of alpha feto protein during gastric-origin secondary cancer of the liver. Presse Med 78: 1277-1278, 1970 (In French).

8. Ishikura H, Fukasawa Y, Ogasawara K, Natori T, Tsukada Y and Aizawa M: An AFP-producing gastric carcinoma with features of hepatic differentiation. A case report. Cancer 56: 840-848, 1985.

9. Liu X, Cheng Y, Sheng W, et al: Analysis of clinicopathologic features and prognostic factors in hepatoid adenocarcinoma of the stomach. Am J Surg Pathol 34: 1465-1471, 2010.

10. Plaza JA, Vitellas K and Frankel WL: Hepatoid adenocarcinoma of the stomach. Ann Diagn Pathol 8: 137-141, 2004.

11. Jo JM, Kim JW, Heo SH, Shin SS, Jeong YY and Hur YH: Hepatic metastases from hepatoid adenocarcinoma of stomach mimicking hepatocellular carcinoma. Clin Mol Hepatol 18: 420-423, 2012.

12. Chang YC, Nagasue N, Abe S, Taniura H, Kumar DD and Nakamura T: Comparison between the clinicopathologic features of AFP-positive and AFP-negative gastric cancers. Am J Gastroenterol 87: 321-325, 1992.
13. Mönch J, Mühler K, Hansen C, et al: The LiverSurgeryTrainer: training of computer-based planning in liver resection surgery. Int J Comput Assist Radiol Surg 8: 809-818, 2013.

14. Gruttadauria S, Marsh JW, Bartlett DL, Gridelli B and Marcos A: Ex situ resection techniques and liver autotransplantation: last resource for otherwise unresectable malignancy. Dig Dis Sci 50: 1829-1835, 2005.

15. Khoury GF, Mann ME, Porot MJ, Abdul-Rasool IH and Busuttil RW: Air embolism associated with veno-venous bypass during orthotopic liver transplantation. Anesthesiology 67: 848-851, 1987.

16. Wen PH, Lin KH, Chen YL, Hsieh CE, Ko CJ and Kuo SJ: Extracorporeal hepatic resection and autotransplantation using temporary portocaval shunt provides an improved solution for conventionally unresectable HCC. Dig Dis Sci 58: 3637-3640, 2013.

17. Zhang KM, Hu XW, Dong JH, et al: Ex-situ liver surgery without veno-venous bypass. World J Gastroenterol 18: 7290-7295, 2012.

18. Gringeri E, Polacco M, D'Amico FE, et al: A new liver autotransplantation technique using subnormothermic machine perfusion for organ preservation in a porcine model. Transplant Proc 43: 997-1000, 2011.

19. Hannoun L, Delrivière L, Gibbs P, Borie D, Vaillant JC and Delva E: Major extended hepatic resections in diseased livers using hypothermic protection: preliminary results from the first 12 patients treated with this new technique. J Am Coll Surg 183: 597-605, 1996.

20. Hatano E, Tanaka A, Shinohara H, et al: Superiority of HTK solution to UW solution for tissue oxygenation in living related liver transplantation. Transplant Proc 28: 1880-1881, 1996.

21. Raab R, Schlitt HJ, Oldhafer KJ, Bornscheuer A, Lang H and Pichlmayr R: Ex-vivo resection techniques in tissue-preserving surgery for liver malignancies. Langenbecks Arch Surg 385: $179-184,2000$. 